|| ISSN(online): 2589-8698 || ISSN(print): 2589-868X ||

International Journal of Medical and Biomedical Studies

Available Online at www.ijmbs.info

PubMed (National Library of Medicine ID: 101738825)

Index Copernicus Value 2018: 75.71

Original Research Article

Volume 3, Issue 11; November: 2019; Page No. 40-46

IJMBS

CLINICAL ASSESSMENT OF SUPRACONDYLAR FEMUR FRACTURE WITH RETROGRADE NAILING IN PATIENTS FROM BIHAR REGION

\title{
Dr. Arun Kumar
}

Assistant Professor, Department of Orthopaedic, Sri Krishna Medical College and Hospital (SKMCH), Muzaffarpur, Bihar, India.

Article Info: Received 04 October 2019; Accepted 30 October. 2019

DOI: https://doi.org/10.32553/ijmbs.v3i11.700

Corresponding author: Dr Arun Kumar

Conflict of interest: No conflict of interest.

\section{Abstract}

A distal femoral fracture is one of the challenging injuries to orthopaedic surgeons. Operative treatment has been the mainstay to obtain early mobilization of patients. Traditionally, stabilization has been achieved with open reduction using several kinds of plates. However, this conventional technique is not a biologic method; it requires large exposure, with its related risk of soft tissue damage, promoting devascularization of bone fragments and compromising fracture healing. [15] Based on above findings the present study was planned to study patient with distal femur fracture including both supracondylar fracture operated with retrograde femoral nail to evaluate the pre and post-surgical outcomes.

The present study was planned in Department of Orthopaedic, Sri Krishna Medical College and Hospital (SKMCH), Muzaffarpur, Bihar, from jan 2018 to December 2018. Total 650 bone injuries were reported in the hospital. From that 15 cases of the supracondylar Fracture Femur were evaluated in the present study.

The data generated from the present study concludes that retrograde intramedullary supracondylar nail is a good fixation system for the distal third femoral fractures, particularly the extra-articular type. The operative time is lessened with decrease in blood loss. Distal screw related local symptoms is a common problem, and is related to the implant and technique. Early surgery, closed reduction, at least two screws in each fragment, and early post-operative knee mobilisation are essential for good union, and good knee range of motion.

Keywords: supracondylar, distal femur, retrograde nailing, biological fixation, femur fracture, etc.

\section{Introduction:}

Supracondylar femur fractures are becoming more common as the population ages. These fractures usually occur in elderly patients with multiple comorbidities and osteoporotic bone; thus, a high rate of complications exists. The goal in treating supracondylar femur fractures, as in treating any periarticular fracture in a weightbearing bone, is restoration of a stable limb for functional, pain-free ambulation. Initially, fixation and, finally, healing of the bone restores stability. Maintaining anatomic alignment and length and preventing stiffness restore function. Avoiding arthritis, which requires restoration of anatomic congruent joint surfaces and maintaining the normal mechanical axis of the limb, prevents pain. Supracondylar femur fractures require anatomically stable internal fixation for best results. Historically, traction achieved adequate results for the treatment of these fractures; however, the outcomes probably would not be considered acceptable today. Maintaining leg length and preventing varus malalignment is difficult with traction. Although surgical risks were avoided, the patient was exposed to the risks of prolonged bedrest, including pulmonary complications, deep venous thrombosis, pressure injuries, disuse osteoporosis, and generalized muscle atrophy and deconditioning. [1]

All current authors agree that the best results are now achieved with operative methods. [2] Involvement of the articular surface demands a congruent anatomic reduction to prevent or minimize posttraumatic arthritis and provide bone stock for later knee replacement or fusion. [3, 4]

Severe comminution often requires fixation of multiple independent fragments with one device to minimize soft-tissue damage. [5] Severely comminuted distal femur fractures are especially 
hard to treat properly. Obtaining adequate fixation may be technically challenging, especially when multiple fragments are present. The significant forces applied to this area, even during restricted patient activities, require a strong implant; however, fixation is difficult because of the wide canal, the thin cortex, and the relatively poor bone quality of the distal femur. [6]

Most surgical failures are caused by inadequate fixation of fracture fragments. Each device has limitations, and no implant can stabilize every fracture type; however, for best results, the device chosen must provide fixation rigid enough for early motion. If comminution and the fracture pattern compromise the use of an implant, the surgeon should be flexible and choose the device that fits best. Supracondylar femur fractures that occur after total knee replacement are also more difficult to treat adequately because the knee replacement prosthesis can interfere with fixation implants. [7]

The distal femur is funnel-shaped, and the area where the stronger diaphyseal bone meets the thinner and weaker metaphyseal bone is prone to fracture with direct or indirect trauma. The surgeon needs to be aware of the shape of the bone when planning surgery so that the implant matches the bone. The approach to the thigh is a standard lateral one, with an incision through the fascia lata and access to the bone along the intermuscular septum under the vastus lateralis. The femoral artery is medial; other neurovascular structures are posterior and thus should not be encountered during surgery.

Supracondylar femur fractures usually occur as a result of low-energy trauma in osteoporotic bone in elderly persons or high-energy trauma in young patients. Fractures proximal to knee replacements may be caused by notching of the anterior cortex when the surgeon placed the prosthesis or may be secondary to the stress riser effect of the interface between the rigid metal and soft bone. $[8,9]$ The treating physician must also be aware of the potential for pathologic fractures through metastatic lesions or primary bone tumors in this area.

With stable fixation, anatomic alignment, and restoration of intra-articular congruency, most patients do well. The more comminuted the fracture and the poorer the quality of bone, fixation, or reduction, the worse the prognosis. Severe comminuted type C3 fractures are expected to develop significant stiffness and posttraumatic arthritis. Patients with open fractures fare worse than those with closed fractures.

Periprosthetic fractures and dementia, heart failure, advanced renal disease, and metastasis lead to reduced survival. Dealying surgery for longer than 4 days leads to increases in 6-month and 1-year mortality. Mortality after native fractures of the distal femur in the geriatric population is high and is comparable to mortality after hip fractures. [10]

Essentially all supracondylar femur fractures require operative intervention because of the severe potential risks of prolonged bed rest. Patients in whom surgery is contraindicated include patients who are bedridden or non-ambulatory with nondisplaced or minimally displaced fractures in which a brace may provide acceptable stability and alignment is not an issue. (Patients with displaced unstable fractures in this group still may require surgery to improve nursing care, decrease pain, and prevent further soft-tissue damage by mobile bone fragments.) Patients with severe life-threatening or other medical problems in which the risks of anesthesia are high may also be treated nonoperatively. Surgical therapy involves reduction followed by fixation to maintain alignment. Options include external fixation and internal fixation. Internal fixation is accomplished by using intramedullary devices (eg, flexible rods, more rigid retrograde or antegrade rods) or extramedullary plates and screws.

All the devices used to stabilize supracondylar femur fractures require exact placement of hardware for optimal rigid fixation. If the coronal and sagittal fracture lines intersect at the entry point of the blade, lag screw, or intramedullary device, fixation is insecure. Placement of the blade within $1.5 \mathrm{~cm}$ of the articular surface is crucial. [11]

The lag screw of the DCS also requires exact placement $2.5 \mathrm{~cm}$ proximal to the articular surface. The screw is $0.5 \mathrm{~mm}$ thicker than the blade and thus must be placed more proximally. With both DCS and blade plates, one or more screws should be placed through the distal holes into the distal fragment for rotational stability. A standard interlocking nail is not feasible with fractures that are too distal $1<7 \mathrm{~cm}$ from the joint) or with displaced intra-articular fractures unless the joint can be reconstructed with cannulated lag screws, because extensive intercondylar comminution prevents rigid fixation with these devices. Supracondylar nails also 
necessitate restoration of the condyles before nail placement. Rush and Zickel devices must be inserted just proximal to the articular surface. These flexible intramedullary devices may not provide adequate fixation. [12]

Use of any plate for supracondylar femur fractures requires strict adherence to AO-ASIF techniques, including interfragmentary compression, indirect reduction, preservation of soft-tissue attachments, and bone grafting. The surgeon must be aware of the trapezoidal shape of the distal femur and align the plate properly. Plates that do not match the metaphyseal flare must be molded to fit. When fixing these complex fractures, the surgeon must choose a device that fits the fracture, rather than try to make the fracture fit the device.

Typically, two distinct mechanisms of injury cause distal femur fractures. In the older population with osteoporotic bone and vulnerable soft-tissue envelope, distal femoral fractures occur predominately after low-energy trauma, e.g., falls and sprain injuries complicated by a high rate of comorbidity (60\% female, older than 60 years). In young patients (60\% male, younger than 40 years), high-energy trauma causes complex injury with comminuted and open fracture pattern. $30 \%$ of patients with distal femur fractures are polytraumatized. [13] $40 \%$ had soft-tissue injuries. $10 \%$ had ligamentous lesions, $8 \%$ had meniscal lesions, $10 \%$ had dissected cartilage fragments and $15 \%$ had patella fractures. $38 \%$ of supracondylar/ intercondylar distal femoral fractures have a coronal plane fracture. [14]

A distal femoral fracture is one of the challenging injuries to orthopaedic surgeons. Operative treatment has been the mainstay to obtain early mobilization of patients. Traditionally, stabilization has been achieved with open reduction using several kinds of plates. However, this conventional technique is not a biologic method; it requires large exposure, with its related risk of soft tissue damage, promoting devascularization of bone fragments and compromising fracture healing. [15] Based on above findings the present study was planned to study patient with distal femur fracture including both supracondylar fracture operated with retrograde femoral nail to evaluate the pre and post-surgical outcomes.

\section{Methodology:}

The present study was planned in Department of Orthopaedic, Sri Krishna Medical College and Hospital (SKMCH), Muzaffarpur, Bihar, jan 2018 to December 2018. Total 650 bone injuries were reported in the hospital. From that 15 cases of the supracondylar Fracture Femur were evaluated in the present study.

All patients were positioned supine with the injured extremity draped free. For the extraarticular fractures, an anterior, midline incision was made that extended from the inferior pole of the patella to the tibial plateau, similar to the approach for intramedullary nailing of the tibia. The patellar tendon was split centrally and retracted to gain access to the intercondylar notch. Using image intensification, either a sharp awl or a 0.25 -inch drill was advanced into the notch, with the knee flexed 30 ${ }^{\circ}$ to $40^{\circ}$, just anterior to the femoral attachment of the posterior cruciate ligament. For the Type C1 intraarticular fractures, a closed reduction and fixation of the condyles with percutaneous, cannulated lag screws placed anterior or posterior to the path of the intramedullary nail was attempted. If reduction of the articular surface was not anatomic, an open reduction through a formal medial parapatellar arthrotomy was performed. All Type C2 and C3 fractures were reduced open. Direct exposure of the intercondylar fracture allowed provisional fixation with Kirschner wires or interfragmentary screws. Once the condyles were reconstructed, a guide wire was advanced past the fracture site into the proximal shaft of the femur. Sequential reaming to 1 to $2 \mathrm{~mm}$ greater than the selected nail was performed and a supracondylar nail that allowed at least 2 bicortical screws to gain purchase in the proximal shaft was placed over the guide wire. The distal tip of the nail was positioned deep to the cortical bone in the notch to prevent impingement on the tibial plateau or patella. The nail was attached to an insertion jig that allowed placement of the interlocking screws proximally and distally through lateral stab incisions. It was imperative to obtain at least 2 screws distally in the condyles to prevent rotation at the fracture site. Accurate measurement of the distal interlocking and lag screws was important to prevent impingement and pain from prominent screws.

All the patients were informed consents. The aim and the objective of the present study were conveyed to 
them. Approval of the institutional ethical committee was taken prior to conduct of this study.

Following was the inclusion and exclusion criteria for the present study.

Inclusion Criteria: Male and female patients more than 18years of age with supracondylar fracture femur with an indication for surgical management even patients with multiple fractures.

Exclusion Criteria: Patients less than 18 years of age, Patients who were non ambulatory or bedridden patients with knee sepsis or Severe infection present somewhere in body, Type IIIB, IIIC compound injuries.

\section{Results \& Discussion:}

Distal femur fractures occur following high-energy impact in young patients often resulting in comminuted and open fractures, whereas low-energy injury is sufficient to cause distal femoral fractures in elderly patients with osteopenic or osteoporotic bone. For the treatment of distal femoral fractures, two major therapeutic principles can be employed: retrograde IM nailing or locking plate osteosynthesis. Both operative stabilizing systems follow the principle of biological osteosynthesis. Protection of soft-tissue envelope due to the minimally invasive approach and closed reduction techniques is better realized using IM. nailing.

Supracondylar fractures occur within the distal $9 \mathrm{~cm}$ of the femur. Fractures were classified according to the AO system, [16] where Type A fractures are extraarticular and Type $\mathrm{C}$ are intraarticular fractures. Fracture types are numbered 1, 2, or 3 based on the degree of comminution: $A 1$ being a simple, 2-part fracture of the metaphysis and $A 3$ having severe comminution. Intraarticular fractures are similarly classified: $\mathrm{C} 1$ fractures are a simple $\mathrm{T}$ or $\mathrm{Y}$ split of the femoral condyles, C2 fractures have metaphyseal comminution, and $\mathrm{C} 3$ have comminution of the articular surface. Open fractures were graded according to the Gustilo and Anderson classification. [17] Open fractures were treated with irrigation and debridement within 6 hours of injury. Fractures were stabilized within 24 hours in most patients. Five patients with multitrauma had their fractures stabilized 2 to 5 days after injury.

Our seri es did not concur with that of Akib et al, which had a mean age of 63 years, against the mean age of 34.2 years. [18] Road traffic accidents accounted for the majority in young population, and male patients, while fall from height was the second most common mode in our series. This series varied from the results seen in a study conducted by Elsoe et al, which had a $61 \%$ incidence as a result of trivial trauma, which can be attributed to increased Road traffic accidents in recent years. [19] Among the various treatment modalities, many studies have been conducted on outcomes of distal femur LCP and supracondylar femoral nail. The obvious advantage of nailing is that it aligns the femoral shaft with condyles reducing the tendency of varus movement at the fracture site. Also, since the bending moment is substantially reduced in nail, so failure of fixation in osteoporotic bone is less. Stabilization of distal femur fractures with periarticular locking plates can cause inconsistent and asymmetric formation of periosteal callus. [20] Significantly less periosteal callus formed in fractures stabilized with locking plates than with IM nails. [21] But most studies conclude that plating and RIMN have similar results in the treatment of extra-articular distal femur fractures. [22-23] Clinical outcome largely depends on surgical technique rather than on the choice of implant. [24] Another study emphasised that cement augmentation and shape memory alloy can also be used for added mechanical stability. This surgical technique is very useful for distal femur fracture with osteoporosis as it promotes fracture healing and early rehabilitation. [25]

Table 1: Sex \& Age Distribution

\begin{tabular}{ll}
\hline Parameters & No. of Cases \\
\hline Sex: & 11 \\
Male & 4 \\
Female & 1 \\
\hline Age: & 2 \\
$20-30$ years & 4 \\
$31-40$ years & 5 \\
$41-50$ years & 3 \\
$51-60$ years & 15 \\
60 years \& above & \\
\hline Total &
\end{tabular}

Table 2: Type of Injury

\begin{tabular}{ll}
\hline Type & No. of Cases \\
\hline Road Accident & 8 \\
\hline Fall from height & 3 \\
\hline Slip and fall & 3 \\
\hline Sports and others & 1 \\
\hline Total & 15 \\
\hline
\end{tabular}


Table 3: Type of Fracture and Duration of Surge

\begin{tabular}{ll}
\hline Side & No. of Cases \\
\hline Right & 10 \\
\hline Left & 5 \\
\hline Total & 15 \\
\hline Type & 5 \\
\hline A1 & 3 \\
\hline A2 & 3 \\
\hline A3 & 1 \\
\hline B2 & 1 \\
\hline C1 & 2 \\
\hline C2 & 15 \\
\hline Total & 3 \\
\hline 30 min to 60 mins & 5 \\
\hline 60 mins to 90 mins & 5 \\
\hline 60 mins to 120 mins & 2 \\
\hline More than 120 mins & 15 \\
\hline Total & \\
\hline
\end{tabular}

Table 4: Time for Radiological union

\begin{tabular}{ll}
\hline Time & No. of Cases \\
\hline $10-12$ weeks & 8 \\
\hline $13-14$ weeks & 4 \\
\hline $15-16$ weeks & 2 \\
\hline $17-18$ weeks & 1 \\
\hline Total & 15 \\
\hline
\end{tabular}

Table 5: Complications and Outcomes

\begin{tabular}{ll}
\hline Time & No. of Cases \\
\hline Anterior knee pain & 1 \\
\hline Knee stiffness & 2 \\
\hline Superficial infection & 1 \\
\hline Delayed union & 1 \\
\hline Shortening & 1 \\
\hline Screw loosening & 1 \\
\hline Total & 7 \\
\hline & \\
\hline Outcomes & 13 \\
\hline Completely Recovered & 2 \\
\hline Medium Recovered & 0 \\
\hline Poor Recovery & 15 \\
\hline Total
\end{tabular}
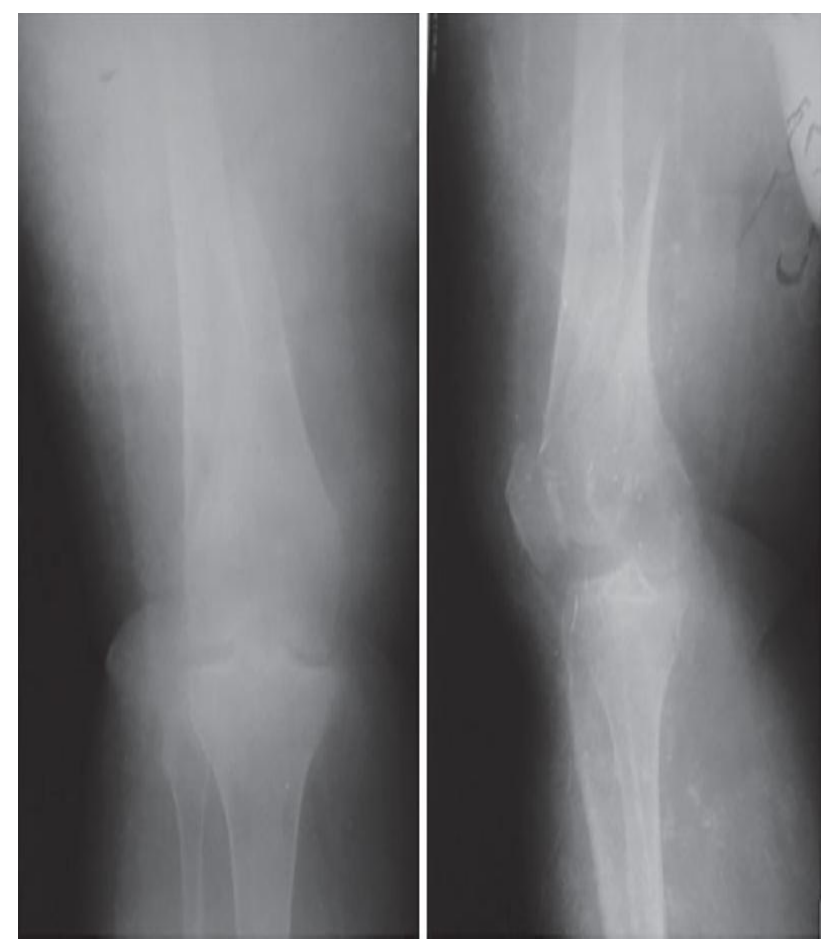

Figure 1: Radiograph of a type A1 supracondylar femoral fracture in male patient. Anteroposterior and lateral view (preoperat ive).

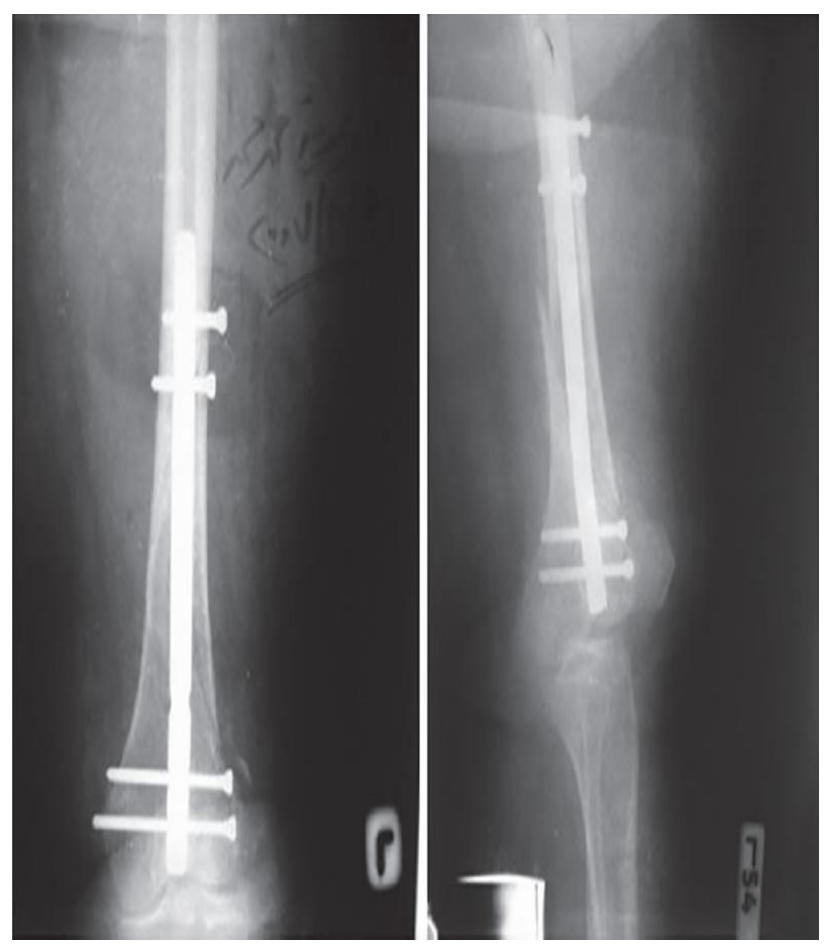

Figure 2: Radiograph of a type A1 supracondylar femoral fracture (anteroposterior and lateral view) fixed with a short retrograde supracondylar femoral nail (postoperative).

A study done earlier [26] showed that the patients with distal femur fracture was associated with extremity injury and other system injury which was 
similar to our study. The mean union time and the mean range of motion was found to be slightly higher in our study when compared to other studies based on the retrograde nailing. [27-28] There was no significant difference in union time and reduction technique between the open and closed fractures. There was no significant difference between the intercondylar fracture and supracondylar fracture in union time and reduction technique. [28-29]

In the past, treatment of distal femur fractures was associated with high complication rates. Although implants and surgical techniques had improved, plate osteosynthesis and IM. nailing suffered from considerable rates of infection, nonunion, and malalignment. Attention to the soft-tissue envelope by introducing the concept of "biological" osteosynthesis and minimally invasive approaches resulted in decreased complication rates. Minimally invasive technique of osteosynthesis can be achieved by using two concepts: minimally invasive plating with an internal fixator - the LISS-DF (less invasive stabilization system - distal femur) - and even more by $\mathrm{RN}$.

\section{Conclusion:}

The data generated from the present study concludes that retrograde intramedullary supracondylar nail is a good fixation system for the distal third femoral fractures, particularly the extra-articular type. The operative time is lessened with decrease in blood loss. Distal screw related local symptoms is a common problem, and is related to the implant and technique. Early surgery, closed reduction, at least two screws in each fragment, and early postoperative knee mobilisation are essential for good union, and good knee range of motion.

\section{References:}

1. Rabin SI, Carlson D, Rabin SL. Complications of Supracondylar Femur Fractures. Loyola U Orthopaedic J. 1995. 4:30-38.

2. Shahcheraghi GH, Doroodchi HR. Supracondylar fracture of the femur: closed or open reduction?. J Trauma. 1993 Apr. 34(4):499-502.

3. Olerud S. Operative treatment of supracondylar-condylar fractures of the femur. Technique and results in fifteen cases. J Bone Joint Surg Am. 1972 Jul. 54(5):1015-32.

4. Schatzker J, Lambert DC. Supracondylar fractures of the femur. Clin Orthop. 1979 Jan-Feb. (138):77-83.

5. Johnson EE. Combined direct and indirect reduction of comminuted four-part intraarticular T-type fractures of the distal femur. Clin Orthop. 1988 Jun. (231):154-62.

6. Pritchett JW. Supracondylar fractures of the femur. Clin Orthop. 1984 Apr. (184):173-7.

7. Herrera DA, Kregor PJ, Cole PA, Levy BA, Jönsson A, Zlowodzki M. Treatment of acute distal femur fractures above a total knee arthroplasty: systematic review of 415 cases (1981-2006). Acta Orthop. 2008 Feb. 79(1):22-7.

8. Yoo JD, Kim NK. Periprosthetic fractures following total knee arthroplasty. Knee Surg Relat Res. 2015 Mar. 27 (1):1-9.

9. McLachlin $\mathrm{S}$, Kreder $\mathrm{H}, \mathrm{Ng} \mathrm{M}$, Jenkinson $\mathrm{R}$, Whyne $\mathrm{C}$, Larouche J. Proximal screw configuration alters peak plate strain without changing construct stiffness in comminuted supracondylar femur fractures. J Orthop Trauma. 2017 Aug 29.

10. Streubel PN, Ricci WM, Wong A, Gardner MJ. Mortality after distal femur fractures in elderly patients. Clin Orthop Relat Res. 2011 Apr. 469(4):1188-96.

11. Schatzker J. Supracondylar fractures of the femur. Schatzker J, Tile M, eds. The Rationale of Operative Fracture Care. 3rd ed. New York: Springer-Verlag; 2005. 409-39.

12. Zickel RE, Hobeika P, Robbins DS. Zickel supracondylar nails for fractures of the distal end of the femur. Clin Orthop. 1986 Nov. (212):79-88.

13. Martinet $O$. The mechanics of internal fixation of fractures of the distal femur: A comparison of the condylar plate (CP) with the condylar screw (DCS). MD Thesis, University of Zurich. 1996.

14. Krettek C, Schandelmeier $P$, Tscherne $H$. Distal femoral fractures. Unfallchirurg 1996;90:2.

15. Thomson $A B$, Driver $R$, Kregor PJ. Long-term functional outcomes after intra-articular distal femur fractures: ORIF versus retrograde intramedullary nail ing. Orthopedics 2008; 31 :748-750.

16. Neer CS, Grantham SA, Shelton ML: Supracondylar fracture of the adult femur. J Bone Joint Surg 49A:591-613, 1967.

17. Gustilo RB, Anderson JT: Prevention of infection in the treatment of one thousand and twenty-five open fractures of long bones. J Bone Joint Surg 58A:453458, 1976.

18. 1. Akib Majed Khan, Quen Oat Tang, Dominic Spicer. The Epidemiology of Adult Distal Femoral Shaft Fractures in a Central London Major Trauma Centre Over Five Years. Open Orthop J. 2017; 11:1277-1291.

19. Elsoe R, Ceccotti AA, Larsen P. Population-based epidemiology and incidence of distal femur fractures. Int Orthop. 2018; 42(1):191-196.

20. Lujan TJ, Henderson CE, Madey SM, Fitzpatrick DC, Marsh JL, Bottlang M. Locked plating of distal femur fractures leads to inconsistent and asymmetric callus formation. J Orthop Trauma. 2010; 24(3):156-62. 
21. Henderson CE, Lujan $T$, Bottlang $M$, Fitzpatrick DC, Madey SM, Marsh JL. Stabilization of distal femur fractures with intramedullary nails and locking plates: differences in callus formation. lowa Orthop J. 2010; 30:61-8.

22. Demirtaş A, Azboy I, Özkul E, Gem M, Alemdar C. Comparison of retrograde intramedullary nailing and bridge plating in the treatment of extra-articular fractures of the distal femur. J Clin Exp Med. 2016; $9(10)$.

23. David SM, Harrow ME, Peindl RD, Frick SL, Kellam JF. Comparative biomechanical analysis of supracondylar femur fracture fixation: locked intramedullary nail versus 95-degree angled plate. J Orthop Trauma. 1997; 11(5):344-50.

24. Hierholzer Christian, Rüden Christian von, Pötzel Tobias, Woltmann Alexander, Bühren Volker. Outcome analysis of retrograde nailing and less invasive stabilization system in distal femoral fractures: A retrospective analysis. Indian J Orthop. 2011; 45(3):243-50.
25. Jihyeung Kim, Seung-Baik Kang, Kyungpyo Nam, Seung Hwan Rhee, Jong Won Won, Hyuk-Soo Han. Retrograde Intramedullary Nailing for Distal Femur Fracture with Osteoporosis. Clin Orthop Surg. 2012; 4(4):307-312.

26. Walling AK. Injuries to the knee ligaments with fractures of the femur The Journal of Bone and Joint Surgery. American Volume. 1982 Dec; 64(9):1324-7.

27. Leggon RE. Retrograde femoral nailing: A focus on the knee. The American Journal of Knee Surgery. 2001; 14(2):109-18.

28. Gellman RE. Treatment of supracondylar femoral fractures with a retrograde intramedullary nail. Clinical Orthopaedics and Related Research. 1996 Nov; (332):90-7.

29. Lucas SE. Intramedullary supracondylar nailing of femoral fractures. A preliminary report of the GSH supracondylar nail. Clinical Orthopaedics and Related Research. 1993 Nov; (296):200-6. 